\title{
Research on the Design of Combination Furniture Based on Toy Brick Style Concept
}

\author{
Liu Zongming, Liu Wenjin \\ Central South University of Forestry and Technology, Hunan, Changsha , 410007, China
}

KEYWORDS: Combination Furniture, Toy brick style, furniture experience

Abstract. In order to better assist the innovative furniture design, toy brick style combination concept is applied to furniture design. This paper analyzes the history and conception of combination furniture based on this concept, and analyzes this concept from game, DIY, green design, function design, and culture, as well as its design style and type, proposes its design method, and verifies the guidance value of toy brick style design concept on design with the design practice on combination furniture for users at different ages as an example.

\section{History and conception of toy brick style combination furniture}

"Toy brick style" combination furniture is a new concept extended by introducing "toy brick style" combination concept into furniture design, and strictly speaking, it belongs to modular combination furniture. The earliest conception of "toy brick style" furniture mainly reflects in children's furniture, which has stronger entertainment, and can combine the parts in different shapes and sizes, without that regular module.

"Toy brick style combination furniture" originates from the ancient Chinese tangram combination furniture, which has roots in ancient Chinese Yanjitu. With continuous development and enrichment in historical evolution, it is widely spread both at home and abroad, and adds interest and meaning to monotonous home life with its uniquely interesting combination mode and flexible and diverse display forms. It breaks normal, formal, and common paradigms with playful (not serious) attitude, graceful charm, and innocent style, and thus to show the fresh and vigorous human life and the wisdom of free creation. The earliest Chinese combination furniture can be traced back to the Yanjitu of Song Dynasty, and gradually it develops into the Diejitu of Ming Dynasty, and evolves into the Qiqiaotu of Qing Dynasty. With historical development, Qiqiaotu evolves into tangram, and is spreading to Japan and Europe soon, therefore a batch of excellent tangram combination furniture emerges both at home and abroad.

The "toy brick style" conception has been seen applied to furniture design in some earlier foreign design works. For example, the massive toy brick style sofa LaMichetta, designed by international furniture design master Mr. GaetanoPesce in 2005, is produced and sold by Italian MeritallaSPa Company, with the sewing method similar to those for antique sofa and the outer mattress. The dimension of toy brick follows certain standard module, which is suitable for industrial production and bears prominent individuality. The shapes can be combined arbitrarily according to the module layout, environment size, and space change; the color can also be combined according to users' preferences; sitting, lying, and leaning can be matched arbitrarily according to the size of human body. In short, any combination is available based on personal preference and size of human body, with people oriented principle, and it fully respects the users' individual needs[1].

Therefore, the so called "toy brick style combination furniture" means that, based on combination furniture, combine the toy brick creativity and connection conception to establish a series of standard modules, and combine these modules into different furniture forms with corresponding connection modes like toy bricks, and thus to meet users'requirements on different space and function." [2]. Therefore, the toy brick style combination furniture possesses 6 features: (1) standard modules (2) interesting toy brick (3) diversified form combinations (4) functional requirements(5) attractive in price and quality(6) green and environmental protection. 


\section{Design concept of toy brick style combination furniture}

Toy brick style combination concept enriches the design connotation of toy brick style combination furniture from the perspective of design idea, after integrating and perfecting its concept, we propose the following "five design concepts", which guide the development and design of "toy brick style" combination furniture:

\section{"Toy brick style" game design concept}

"Toy brick style" creativity is inspired from children's "toy brick" with predecessors of the wooden toys in ancient China, such as "burr puzzle" and "tangram". The "toy brick style" combination furniture is mostly featured that the parts also belong to products, which consist of many "toy brick" units and they are combined into different shapes. Especially with the development of children's "toy brick style" combination furniture, more fun of "playing" is shown, as well as functionality and practicability.

\section{"Toy brick style" DIY design concept}

DIY is the abbreviation of DO IT YOURSELF, and this design has been largely developed abroad, for example, the furniture design of Ikea is developed by following this design concept. DIY design concept mainly has two application modes in furniture design, one is DIY in the whole process from furniture design to material selection, processing, making, and painting, and this kind of DIY furniture making requires higher technical level of furniture maker; the other DIY modular furniture adopts the semi-finished product (unit form and assembly of certain modules), produced by furniture manufacturer, after purchasing it, customers assemble and design it as required by their own environment[1]. This paper discusses the latter "toy brick style" combination furniture, which allows consumers to directly participate in design of furniture style.

\section{"Toy brick style" green design concept}

"Toy brick style" combination furniture follows the principle of combination and shaping with the same or similar single part, which is an independent product, and any broken single part will not affect the integrated combination function of the furniture. Therefore, this kind of furniture realizes industrial mass production mode and reflects the real "excellent quality and reasonable price", which are the real feedbacks of green design concept.

\section{"Toy brick style" function design concept}

"Toy brick style" combination furniture has quite flexible function, which can be realized by combining into different forms according to different needs of consumers. Used indoor and outdoor, its feature "in line with local conditions" can be largely reflected. It can separate each functional space, bring optional "bionic" shaping, and even make full use of each dead space, to realize diversified functions and achieve space-saving, flexible and free use results.

\section{"Toy brick style" culture concept}

Jean Baudrillard points out in La société de consummation, "Nowadays, few objects will be provided without reflecting its background. The relation between consumer and object changes consequently, he will not just view an object from its special use but view the full set of the object from its whole meaning. " Therefore, as a consumption product, the "object" reflects more of its difference with other products than its materiality. When selecting a product, consumers not only select its "expendability", but also make "self-actualization" through it. So the product not only meets the functionality, but also shows the implicit factors such as class, taste, and cultural psychology of its owner. The individualized and interesting feature of "toy brick style" combination furniture is bound to attract the consumers who love such consumption symbols [3].

\section{Design style of toy brick style combination furniture}

The combination form of toy brick style combination furniture is influenced more by its own connection structure than its functional and aesthetic requirements, material and color. The way for various parts in furniture to be connected and functioned by certain use functions is called the structure, which is the main part of furniture and the basic guarantee to realize function. Structural form depends on the shaping, use function, material feature, and the possibility of processing 
technology, and it includes the internal and external structures of furniture. The internal structure of furniture is a certain combination mode between parts, and it depends on the changes of material and development of science and technology, for example, wooden furniture, metal furniture, plastic furniture, and vine furniture and other furniture have their own structural features. The external structure of furniture directly contacts users and directly reflects its appearance shaping, so it must match users in dimension, proportion, and shape. For example, the seat surface must have proper height, depth and back angle, so that the chair can relieve people's fatigue; while the storage-type furniture needs to match the size of the articles to be stored, on condition that it enables users to have an easy access to their storage. The external furniture structure designed by this requirement also lays a foundation for furniture's aesthetic function requirements.

Based on whether the single part has connection structure, toy brick style combination furniture can be divided into toy brick style combination furniture with connection structure and toy brick style combination furniture without connection structure, and they have the following main features:

\section{Toy brick style combination furniture without connection structure}

For toy brick style combination furniture without connection structure, its units have independent functions and are directly combined just like toy bricks, and they can be piled and shaped (as shown in Figure 1) at will. The units can be identical, in standard geometric shape or organic shapes different from each other, but without any connection structure between them, therefore the combination forms between them are random and free with multiple choices. Any broken unit will not affect the integral shape, besides, this kind of furniture can select color scheme for its units freely according to its own environment.
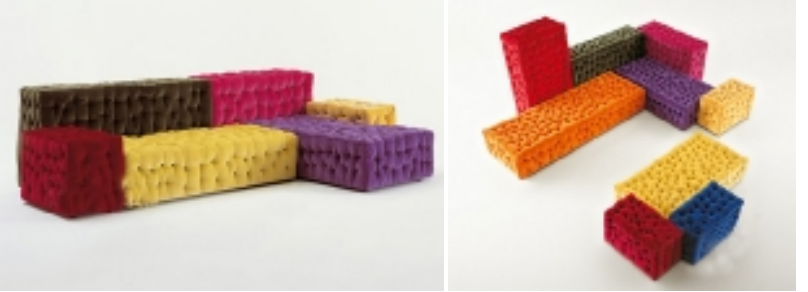

Figure 1 Toy brick style sofa (LaMichetta)

\section{Toy brick style combination furniture with connection structure}

For this kind of furniture, its units also have their own independent functions and are combined in an associated way by a certain rule to form a new shape with complete functions, which is toy brick style combination furniture with connection structure. While this rule can be like the mortise and tenon joint in Ming-style furniture or like the "burr puzzle" style self-locking structure (as shown in Figure 2) of the ancient Chinese wooden toy.

As shown in Figure 3, the "black \& white chequered" combination book shelf adopts common use form of mortise and tenon in design, innovates in appearance and combination, and highlights the functionality and sense of form. Each unit is connected by mortise and tenon joint, and the book shelf as a whole forms many styles (as structure and the decoration node) due to different structures, and can be combined into new shapes at will.

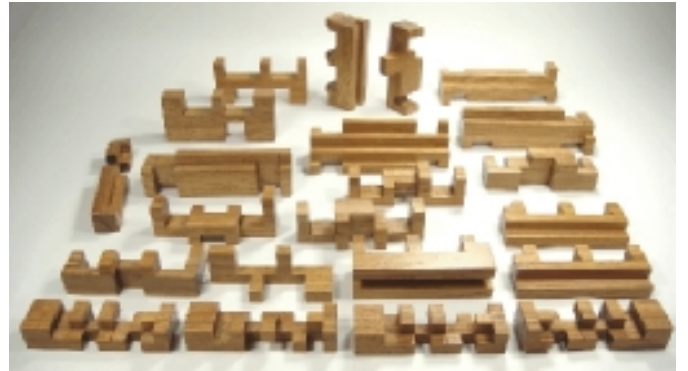

Figure 2 "Burr Puzzle" structure 


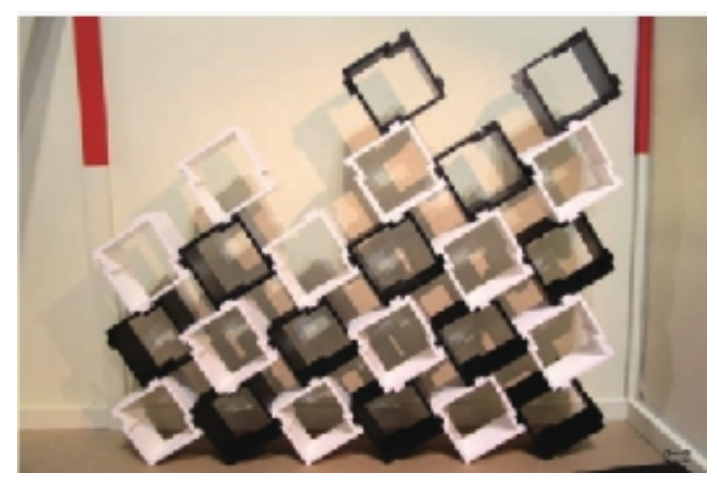

Figure3 "Black \& white chequered" combination book shelf

\section{Design methods for toy brick style combination furniture}

\section{Rational shaping method}

Rational shaping method starts from modern rational aesthetics, and plans for furniture shaping mainly with pure geometric shapes. Any method following rational principle in shaping can be classified into rational shaping method; guided by this concept, whether abstract or concrete shaping is adopted in furniture shaping, as long as the shaping is made or displayed in line with rational consciousness, it has the features of rational shaping. In principle, rational shaping methods mainly adopt geometric shapes, while the decoration part can adopt different geometric shapes as needed. When treating the same space or same group of furniture shaping with same or similar geometric shapes repeatedly, the full set of furniture will certainly achieve the full, harmonious, and unified result [4].

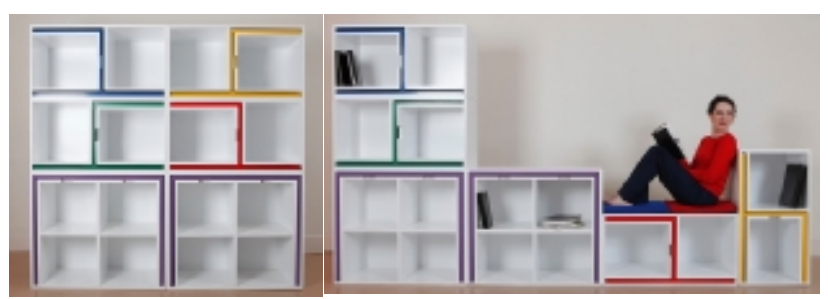

Figure 4 Colorful combination book shelf

In general, most structured unit shaping of toy brick style combination furniture is realized by rational shaping method, for example, triangle, rectangle, and other polygons are referred. See the rectangle combination furniture as shown in Figure 4.

\section{Sensible shaping method}

Sensible shaping method mainly adopts the free form that is rich in sensible idea for furniture shaping design, based on modern sensible aesthetics. Shaping is conceived by the image emerged in one's consciousness, while the rich influence is brought by keen shaping sense and often becomes the impromptu product by accident. Sensible shaping fully relies on brainstorming, and it fully catches the shaping elements occurred randomly in brain to integrate them sensibly, so the final shaping is random and accidental. In this way, we get the toy brick style combination units, with shaping closer to organic form, so it belongs to a shaping method of returning to nature and harmony (as shown in Figure 5).

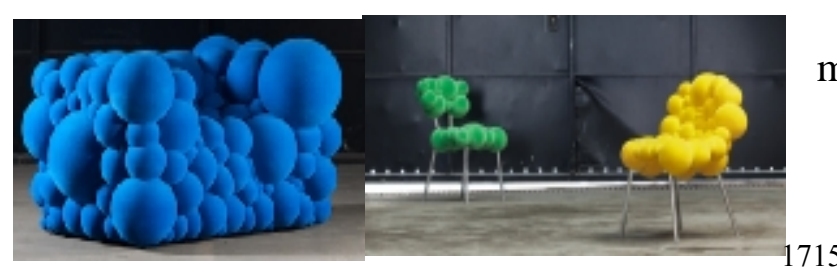

Figure 5 Furniture with sensible shaping method 


\section{Traditional shaping method}

After observation and appreciation of traditional furniture, and learning about the changes of furniture shaping design from the past to today, we can conclude certain methods and design direction of modern shaping in the future. Similarly, by observing the trend of shaping form, we can find many techniques of traditional and currently popular shaping, and by bearing these in mind, we will make more breakthroughs in design innovation of the modern furniture shaping. As shown in Figure 6, the design inspired by the coin shape of Chu State, reflects the shaping method of returning to tradition.

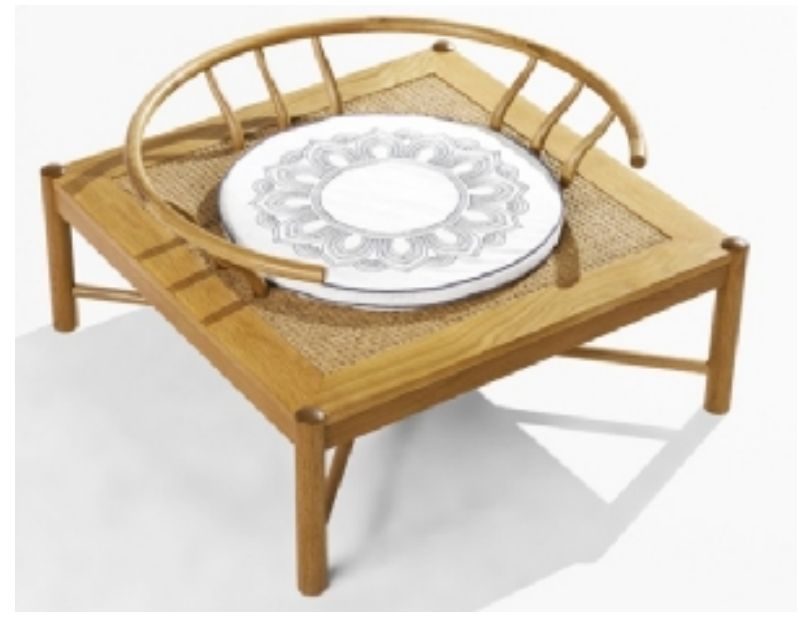

Figure 6 Furniture with traditional shaping method

\section{Design practice of toy brick concept on furniture}

In design practice, as the extension of human body function, furniture should be designed in accordance with users'cognition and behavior needs in different stages, to standardize and please user behavior, and thus to make user grow in the process of furniture experience, while the design concept of toy brick style combination furniture exactly meets such need. In the process of design, start from the design concepts of toy brick style combination including game, DIY, function design, green design, user's culture, adopt the style without structural connection to integrate and innovate user behaviors in different stages, and design a combination furniture form that can meet the demands in multiple stages. As shown in Figure 7, this design captures the features of growth and development of children in childhood to design the functional forms for children to climb, get in, slide, shake, paint, and learn, to make them grow in happy experiences. At the same time, as shown in Figure 6, the combination furniture designed by integrating user's behaviors in their childhood and youth stage, adopts the same parts and structure-free connection mode to allow user to combine into multiple forms as needed, and allow user to not only experience its growable functions, but also obtain its longer service life. 


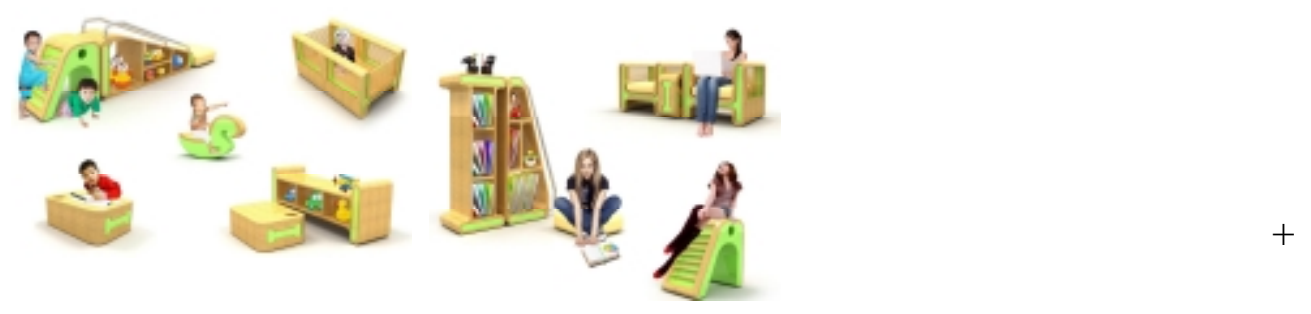

Figure 7 Growable combination furniture (design by Liu Zongming )

\section{Conclusion}

To further help designers to innovate furniture design, in this paper, it studies the history and conception, analyzes the five design concepts, summarizes and concludes the innovative design styles, and proposes the innovative design methods of toy brick style combination furniture. At last, it takes the design practice on combination furniture suitable for different ages as an example to verify the guidance value of toy brick style design concept on furniture design. Meanwhile, its research results may also guide the innovative design of other daily products.

\section{Acknowledgment}

We should be thankful for a lot for the support from Fund project 1 of Scientific Research key Project of the Education Department of Hunan Province in 2014: Theoretical research on the development of children's furniture Based on the idea of saving (Project No. 14A041) and Fund project 2 of Philosophy and Social Science Fund Supported Project of Hunan Province in 2013: research on product innovation design based on growable-type design method (No. 13YBA114);

\section{References}

[1] Sun Yuanyuan. Research on the Application of DIY Concept in the Design of Modular Furniture [D]. Jiangnan University, 2007: P23.

[2] Wei Jianyuan, Li Cisheng. Analysis on the Design of Toy Brick Style Combination Furniture [J]. Guangxi Journal of Light Industry, 2009 (01): P50.

[3] Wei Jianyuan, Li Cisheng. Research on the Design of Toy Brick Style Product Meeting Individual Requirements of User [J]. Art and Design, 2010 (01): P35.

[4] Cui Chunjing. Research on the Essential Elements that Influence the Design of Modern Furniture Form [D]. Qilu University of Technology(former Shandong University of Technology ), 2008. 